\title{
Influence of hand position on the near effect in 3-D attention
}

\author{
Petra M. J. Pollux and Patrick A. Bourke \\ University of Lincoln, Lincoln, England
}

\begin{abstract}
Voluntary reorienting of attention in real depth situations is characterized by an attentional bias to locations nearer the viewer once attention is deployed to a spatially cued object in depth. Previously, this effect (initially referred to as the near effect) was attributed to access of a 3-D viewer-centered spatial representation for guiding attention in 3-D space. The aim of this study was to investigate whether the near effect could have been associated with the position of the response hand, which was always near the viewer in previous studies that investigated endogenous attentional shifts in real depth. In Experiment 1, the response hand was placed at either the near or far target depth in a depth-cuing task. Placing the response hand at the far target depth abolished the near effect, but failed to bias spatial attention to the far location. Experiment 2 showed that the response hand effect was not modulated by the presence of an additional (passive) hand, whereas Experiment 3 confirmed that attentional prioritization of the passive hand was not masked by the influence of the responding hand on spatial attention in Experiment 2. The pattern of results is most consistent with the idea that response preparation can modulate spatial attention within a $3-\mathrm{D}$ viewer-centered spatial representation.
\end{abstract}

Over the past 20 years, evidence for a depth-aware representation underlying spatial attention has accumulated. Studies investigating the nature of selective attention in 3-D space have reported reliable effects of location cues to near and far locations on performance measures in real depth situations (Couyoumdjian, Di Nocera, \& Ferlazzo, 2003; Downing \& Pinker, 1985; Gawryszewski, Riggio, Rizzolatti, \& Umiltà, 1987), in stereoscopic displays (e.g., Atchley \& Kramer, 1998; Atchley, Kramer, Andersen, \& Theeuwes, 1997; Bourke, Partridge, \& Pollux, 2006; Theeuwes, Atchley, \& Kramer, 1998), in perceived space (Han, Wan, \& Humphreys, 2005), and within pictorial scenes (Parks \& Corballis, 2006). Particularly in real depth experimental situations, deployment of attention is characterized by an asymmetric effect of spatial cuing, first referred to as the near effect by Gawryszewski et al. in 1987. In their study, spatial attention was cued at fixation with high probability to one of two target LEDs, one located near the viewer and the second at a depth beyond fixation, but still within reach. Their results showed that detection times were greater when the target was presented at the uncued location, but also that this effect of cuing varied with direction. Detection times for targets at the far location when attention had been cued to the near LED were greater than when attention had been cued to the far location and the target was presented at the near LED (Gawryszewski et al., 1987). Comparable asymmetric effects have been observed within pictorial scenes (Parks \& Corballis, 2006) and in stereoscopic depth, where reorienting from near to far locations is associ- ated with greater error costs (Atchley et al., 1997; but see Atchley \& Kramer, 1998, for an exception). A few studies have shown that the distribution of attention in depth is characterized by a gradient that is maximal at attentional focus and declines at more peripheral locations (Andersen, 1990; Downing \& Pinker, 1985). Andersen presented random-dot stereograms of horizontal or vertical bars at the center with distractor bars presented at either the same or different depths as the center bars, and found that performance declined as a function of distance from attentional focus. Downing and Pinker also showed that the cost of reorienting to stimuli at uncued locations in real space (varying in horizontal distance and in depth) was greater when the uncued target was at a different depth. Their analysis of reorienting cost in relation to distance from attentional focus further showed that attention seems to decline as a function of a gradient defined in terms of visual-angle separation, and that this decline is stronger for stimuli presented at a depth different from that of the attentional focus.

The number of studies reporting the near effect is increasing, but little is known about the processes underlying the effect. In Gawryszewski et al.'s (1987) original explanation, the near effect was associated with a viewercentered spatial representation accessed for guiding attention in 3-D space. They proposed that an attentional space is dynamically created between the attended object and the viewer, in response to the spatial depth cue. More specifically, Gawryszewski et al. argued that when attention is cued to the near location, attention spreads from

P. M. J. Pollux, ppollux@lincoln.ac.uk 
the maximal focus of attention to the observer. As a result, spatial awareness is enhanced at the near (relative to the far) location when the target is presented in this condition. When attention is cued to the far location, the attentional space extends from the far location to the body, resulting in equal spatial awareness of the near and far LED when the target is presented. Consequently, reorienting attention from the cued near location to the far target LED is relatively slower (associated with longer target detection times) than reorienting from the cued far location to the target near the viewer.

The idea that the near effect may reflect a general attentional bias toward near stimuli is not supported by existing findings, because detection times to near and far targets are generally comparable when the cue predicts the target location correctly in real depth situations (Couyoumdjian et al., 2003; Gawryszewski et al., 1987). Couyoumdjian et al. further showed that the near effect seems to be independent of the boundary between peripersonal and extrapersonal space. Although the authors found that cuing effects on target detection times were greater in a condition where cued and target locations were separated by the boundary between peripersonal and extrapersonal space ( $1 \mathrm{~m})$, as compared with the situation where both cued and target locations were within either peripersonal or extrapersonal space, this boundary effect did not interact with reorienting direction. A comparable near effect was observed across all conditions, suggesting that the reorienting bias is independent of the proposed functional specialization of 3-D space (Couyoumdjian et al., 2003; Previc, 1998).

Although explanations to date assume that the near effect is associated with access to a viewer-centered representation for guiding attention in 3-D space (e.g., Atchley et al., 1997; Downing \& Pinker, 1985; Gawryszewski et al., 1987; Parks \& Corballis, 2006), converging evidence suggests that the distribution of spatial attention in peripersonal space can be influenced by the position of the viewer's hand when he or she performs tasks involving manual responses (e.g., Eimer, Forster, Van Velzen, \& Prabhu, 2005; Eimer \& Van Velzen, 2006; Mattingley, Robertson \& Driver, 1998). In a neuropsychological investigation of visual extinction, Mattingley et al. showed, for example, that extinction of stimuli within left space was reduced when stimulus presentation was initiated by a keypress with the patient's left hand. ERP findings reported by Eimer et al. (2005) showed that when one hand was cued for responding (lift of index finger), somatosensory ERP components elicited by (task-irrelevant) tactile stimuli presented between cue onset and a go/no-go signal were enhanced. Both findings were considered consistent with the idea that preparation of manual responses is associated with shifts of attention to the response hand (Eimer et al., 2005; Eimer \& Van Velzen, 2006) or with enhanced spatial awareness within the visual field in which the response hand is located (Mattingley et al., 1998).

A different line of evidence suggests that under certain task conditions, attention is prioritized to the location of the viewer's hand, even when this hand is not used for responding (Reed, Grubb, \& Steele, 2006). Reed et al. used a horizontal cuing task (presented on a computer screen) with highly predictive cues for target location. Participants were instructed to place one hand in close proximity to one of the two possible target locations while responding with the other hand (positioned away from the screen) at detection of the targets. Across five experiments, detection times to targets presented close to the passive hand were found to be faster than those to targets presented at the alternative location, independent from cue validity. This attentional prioritization effect persisted when the hand was hidden from view and when it was replaced with a rubber glove, but was absent when it was replaced with a visual anchor. It appears that proprioceptive information received from the hand is sufficient for attentional prioritization of space around the hand, but visual information is not sufficient unless a connection is made between the visual input and the actual hand. Reed et al. further showed that the extent of the prioritized area around the passive hand was relatively small, because the effect disappeared when the hand was placed toward the side of the computer screen, increasing the horizontal distance between the stimulus and the hand to $11 \mathrm{~cm}$. The consistency between this pattern of results and characteristics of bimodal neurons in peripersonal space (see, e.g., Graziano \& Gross, 1995 ) suggests that the attentional prioritization effect is likely to reflect the influence of bimodal hand-centered representations on spatial attention (Reed et al., 2006).

The accumulating evidence for hand-centered attention in peripersonal space raises the question of whether allocation of spatial attention in the depth-cuing task is influenced by the position of the response hand. Because the hand was always positioned near the viewer in previous studies investigating attention in real depth situations, the near effect could have been associated with a bias to the response hand instead of a bias to viewer-centered near space. In the present study, we aim to investigate this question by varying the position of the responding hand while the participants perform the depth-cuing task. In Experiment 1 , participants placed their response hand at the same depth of either a near or far target LED. If the near bias in the reorienting of attention reflects a bias toward the response hand, placing the hand at the same depth as the far LED may reverse the reorienting bias to the far target location. Alternatively, if the near effect reflects the influence of a 3-D viewer-centered spatial representation on spatial attention, as suggested by Gawryszewski et al. (1987), the bias should be unaffected by response hand position.

\section{EXPERIMENT 1}

\section{Method}

Participants. Twenty-eight volunteers participated in Experiment $1 ; 14$ were allocated to the hand-far condition (mean age $=$ 24 years, $S D=5.6$ ) and 14 to the hand-near condition (mean age $=$ 23 years, $S D=4.5$ ). All participants were right-handed, had normal or corrected-to-normal vision, and reported no problems of color vision or depth perception.

Apparatus and Stimuli. The participants were seated in a comfortable chair. Each participant's head was held steady with a chinrest, and each looked down a rail at three LEDs mounted on rods. Distances of the near, middle, and far LEDs from the viewer's posi- 


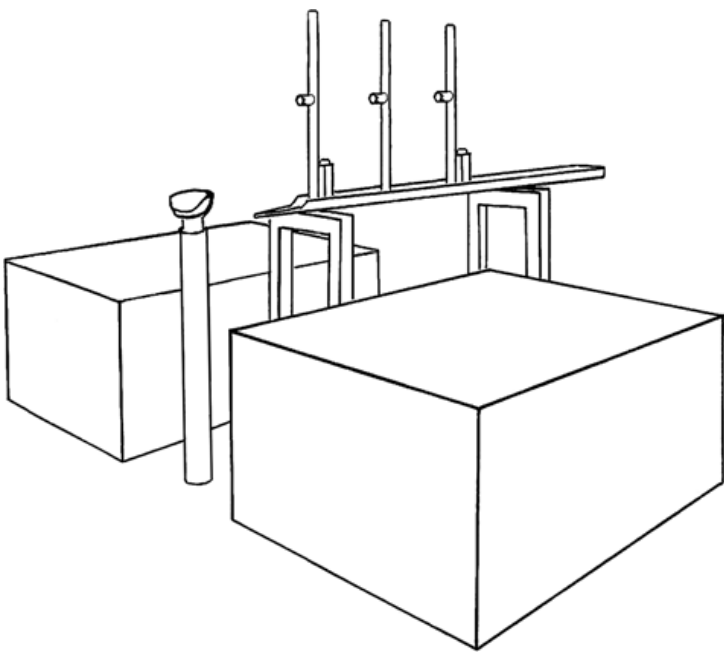

Figure 1. Schematic illustration of the experimental setup. See the text for further explanation of the variations for each experiment.

tion were 19, 31, and $43 \mathrm{~cm}$, respectively (see Figure 1). All testing took place in a fully lit room under binocular viewing conditions, and participants were instructed to maintain fixation on the center LED. To maximize control over luminance differences among the LEDs, a small cap was placed on each light; a hole had been drilled in the middle of the front face of the cap. The diameter of this hole was $2 \mathrm{~mm}$ for all three caps (visual angle was $0.6^{\circ}$ and $0.27^{\circ}$ for the near and far target stimuli, respectively). Luminance of the LEDs was measured with a photometer (Hagner Universal, Model S3) positioned on a tripod at a distance of $30 \mathrm{~cm}$ from the LED. Luminance levels were adjusted to approximately $21 \mathrm{~cd} / \mathrm{m}^{2}$ for the two red target LEDs (with the caps on) and to $58 \mathrm{~cd} / \mathrm{m}^{2}$ for the colors green and blue at the center LED.

To ensure that the LEDs were viewed at eye level, an eye level marker was constructed, consisting of a transparent plastic square fixed to two rods, both attached to a base. A horizontal line was marked across the plastic square. This marker was placed between the chinrest and the LEDs. The chinrest and chair were adjusted until participants reported that the marker line crossed the midlines of all three LEDs. Participants were subsequently asked to describe the visual experience associated with fixating at the center LED All participants reported symmetric diplopic images of the near and the far LED.

Procedure. For the experimental tasks, the participants were required to respond to the onset of a red target light presented at the near or far location by pressing a key on a buttonbox affixed to the rail behind the far rod in the hand-far condition and behind the near rod in the hand-near condition. The hand was placed around the buttonbox and the rod: The thumb was placed to the front of the rod, the index finger (used for responding) was placed on the response key, and the remaining three fingers were placed around the back of the buttonbox, resting against the box and on the rail. The distance between the response key and the LED was $9 \mathrm{~cm}$. Two cardboard boxes were fixed to the table to ensure comfortable positioning of the arms. Participants were instructed to place their left (nonresponding) arm on the left box (placed away from the target scene) and to refrain from moving this arm beyond the depth of fixation. Both arms were visible for the duration of the experiment.

The probable location of the target was cued by lights presented at the central LED (green or blue). The cue light was presented until the target appeared, and targets were presented for $70 \mathrm{msec}$. In the depth-cuing task, in both groups of 14 people, 7 participants were instructed to attend near in response to the blue cue light, and to attend far in response to the green cue light. Cue-location mapping was reversed for the remaining 7 participants within each group. The probability of valid versus invalid cued trials was 80:20, and the task consisted of four blocks of 240 trials. Each block consisted of 160 valid cued trials, 40 invalid cued trials, and 40 catch trials. These catch trials were included to prevent anticipatory responses. A neutral cuing condition was not included, given the concerns raised about the use of this condition as a baseline for measures of facilitation and inhibition in covert visual spatial orienting tasks (Gawryszewski et al., 1987; Jonides \& Mack, 1984). Because previous findings showing that effects of endogenous horizontal cuing on detection times tend to reach a maximum around $300 \mathrm{msec}$ after cue onset (e.g., Müller \& Rabbitt, 1989), stimulus onset asynchrony (SOA) was varied randomly at either 300 or $600 \mathrm{msec}$.

Since the retinal regions covered by the near and far target lights were not the same, we measured detection times in a control task (target-control task) in which red lights illuminated at random at the two target locations. Participants were instructed to respond as quickly as possible on detection of the red light while maintaining gaze at the center LED. A warning tone was presented 300 or $600 \mathrm{msec}$ before presentation of the red light. Delay between response (or the 1,000-msec cutoff in the case of catch trials) and onset of the cue was $500 \mathrm{msec}$. The same task parameters were used to measure detection of the cue lights in a second control task (cuecontrol task). Both tasks consisted of 112 trials (in addition to 16 catch trials, in which the warning tone was not followed by a light).

Participants were introduced to the depth-cuing task in two practice blocks, each consisting of 40 trials. To allow familiarization with the cue-color/location mapping, the first practice block consisted of valid cued trials only. The second block consisted of 32 valid, 8 invalid, and 8 catch trials. Experimental blocks of trials were paused automatically after every 40 trials for a time determined by the participant. Errors (RT $\leq 100 \mathrm{msec}$ or $\geq 1,000 \mathrm{msec}$, and responses on catch trials) were repeated after each block of 240 trials. Control tasks were presented at the end of the testing session.

To be able to exclude trials in which eye movements were made between the onset of the cue light and the offset of the target light, horizontal and vertical electrooculograms (HEOGs and VEOGs, respectively) were recorded with $\mathrm{Ag} / \mathrm{AgCl}$ electrodes placed on the outer canthi of the left and right eyes, and below and above the left eye, referenced to two mastoid electrodes placed behind the two ears. Each EOG was digitized at $256 \mathrm{~Hz}$ per channel and amplified with a bandpass of $0.1-35 \mathrm{~Hz}$. To establish whether eye movements could be detected in the EOGs, a short test was developed consisting of 40 trials. Participants were requested to move their eyes (in blocks of 5 trials) to the near and far LEDs. Each trial was initiated by a warning tone. Testing on 4 participants revealed clearly detectable waves in response to horizontal eye movements and divergent/ convergent eye movements on all 40 trials when the signals from the electrodes placed around the eyes were compared with signals from mastoids (below the eyes for VEOGs, outer canthi for HEOGs). All trials in which the amplitude exceeded $70 \mu \mathrm{V}$ between cue onset and target onset were tagged during testing. Final rejection of eye movement trials occurred off-line after testing by manual inspection of all tagged trials.

\section{Results and Discussion}

Depth-cuing task. Error trials were excluded from RT analysis of the depth-cuing task. Means of median RTs were entered in a mixed, repeated measures ANOVA with the factors SOA, target location (near, far), and cue (valid, invalid). Response hand position (RHP; near, far) was entered as the between-participants factor. Only significant effects are reported here (Table 1 shows the results for all effects in the depth-cuing task). The analysis revealed a significant main effect of target location $[F(1,26)=$ 
Table 1

ANOVA Results of Detection Time in the Depth-Cuing Task in Experiments 1-3

\begin{tabular}{|c|c|c|}
\hline Effect & $F$ & $p$ \\
\hline \multicolumn{3}{|l|}{ Experiment 1} \\
\hline Response hand position (RHP) & $<1$ & \\
\hline Stimulus onset asynchrony (SOA) & 6.06 & .021 \\
\hline Target location & 71.58 & $<.001$ \\
\hline Cue & 54.57 & $<.001$ \\
\hline $\mathrm{SOA} \times \mathrm{RHP}$ & $<1$ & \\
\hline SOA $\times$ target location & 1.74 & .19 \\
\hline SOA $\times$ cue & 38.16 & $<.001$ \\
\hline Cue $\times$ RHP & 2.47 & .13 \\
\hline Target location $\times$ RHP & 1.93 & .17 \\
\hline Target location $\times$ cue & 10.73 & .003 \\
\hline Target location $\times$ cue $\times$ RHP & 5.72 & .024 \\
\hline $\mathrm{SOA} \times$ cue $\times \mathrm{RHP}$ & 2.1 & .15 \\
\hline SOA $\times$ target location $\times$ RHP & $<1$ & \\
\hline SOA $\times$ target location $\times$ cue & $<1$ & \\
\hline SOA $\times$ target location $\times$ cue $\times$ RHP & $<1$ & \\
\hline \multicolumn{3}{|l|}{ Experiment 2} \\
\hline RHP & $<1$ & \\
\hline Hand & $<1$ & \\
\hline Target location & 32.17 & $<.001$ \\
\hline Cue & 26.71 & $<.001$ \\
\hline Hand $\times$ RHP & $<1$ & \\
\hline Hand $\times$ target location & $<1$ & \\
\hline Hand $\times$ cue & $<1$ & \\
\hline Cue $\times$ RHP & $<1$ & \\
\hline Target location $\times$ RHP & 9.17 & .009 \\
\hline Target location $\times$ cue & 8.46 & .011 \\
\hline Target location $\times$ cue $\times$ RHP & 8.39 & .012 \\
\hline Hand $\times$ cue $\times$ RHP & $<1$ & \\
\hline Hand $\times$ target location $\times$ RHP & $<1$ & \\
\hline Hand $\times$ target location $\times$ cue & $<1$ & \\
\hline Hand $\times$ target location $\times$ cue $\times$ RHP & 1.63 & .22 \\
\hline \multicolumn{3}{|l|}{ Experiment 3} \\
\hline Passive hand position (PHP) & $<1$ & \\
\hline Hand & 1.35 & .26 \\
\hline Target location & 41.54 & $<.001$ \\
\hline Cue & 65.96 & $<.001$ \\
\hline Hand $\times$ PHP & $<1$ & \\
\hline Hand $\times$ target location & $<1$ & \\
\hline Hand $\times$ cue & 2.86 & .11 \\
\hline Cue $\times$ PHP & $<1$ & \\
\hline Target location $\times$ PHP & $<1$ & \\
\hline Target location $\times$ cue & 23.76 & $<.001$ \\
\hline Target location $\times$ cue $\times$ PHP & $<1$ & \\
\hline Hand $\times$ cue $\times$ PHP & $<1$ & \\
\hline Hand $\times$ target location $\times$ PHP & 1.9 & .19 \\
\hline Hand $\times$ target location $\times$ cue & $<1$ & \\
\hline Hand $\times$ target location $\times$ cue $\times$ PHP & 2.6 & .09 \\
\hline
\end{tabular}

71.58, $p<.001]$, with longer RTs to targets presented at the far target location. The main effect of cue was also significant $[F(1,26)=54.57, p<.001]$, reflected in longer RTs to invalid cued than to valid cued targets. The effect of cuing was greater at the far than at the near target location, indicated by the significant interaction between cue and target location $[F(1,26)=10.73, p=.003]$. Post hoc analysis (Bonferroni corrected) showed that the effect of cue was significant at both target locations ( $p<.001$, for both comparisons) and that the effect of target location was significant in both cue conditions $(p<.001$, for both comparisons). To allow a comparison between cuing effects at the near and far target locations, difference RT values due to cuing (RT invalid - RT valid) were calculated. Comparison of these difference values confirmed that the effect of cuing was significantly greater at the far than at the near $[t(27)=3.02, p=.005]$ target location. More important, however, was the significant interaction among cue, target location, and RHP $[F(1,26)=5.72, p=$ .024]. Figure 2 shows that the cuing effect in the RH-near condition is asymmetric; this asymmetry is absent in the $\mathrm{RH}$-far condition. Post hoc analysis showed that this result was due to the effect of RHP on detection times to invalid cued targets at the far target location. RTs in this condition were significantly longer in the RH-near condition than in the RH-far condition ( $p=.013$ ), whereas RTs to invalid cued targets at the near location were not significantly different in either response hand condition $(p=.26)$. The difference between the RH-near and $\mathrm{RH}$-far conditions was also not significant for valid trials (valid-near, $p=$ .44 ; valid-far, $p=.36$ ). The effect of SOA was significant, with longer RTs at SOA $=300[F(1,26)=6.06$, $p=.021]$, but this effect was different for the valid and invalid [SOA $\times$ cue: $F(1,26)=38.16, p<.001]$ cue conditions. Post hoc analysis showed that the effect of SOA was observable on valid trials, but not on invalid trials. In the valid cue condition, RT at SOA $=300$ were significantly longer than at SOA $=600(p=.006)$, whereas this difference was not significant on invalid trials $(p=.53)$, resulting in enhanced cuing effects at $\mathrm{SOA}=600$.

Control tasks. For analysis of the target control task, means of median RTs were analyzed with the factors target location and RHP. The main effect of RHP was not significant $[F(1,26)=1.02, p=.32]$, but RTs to lights presented at the far location were significantly longer than RTs to near targets $[F(1,26)=24.2, p<.001]$. This effect of target location was similar for both RHP conditions $(F<1)$. Means of median RTs to near and far targets were $261 \mathrm{msec}(S E=7)$ and $276 \mathrm{msec}(S E=8)$, respec-

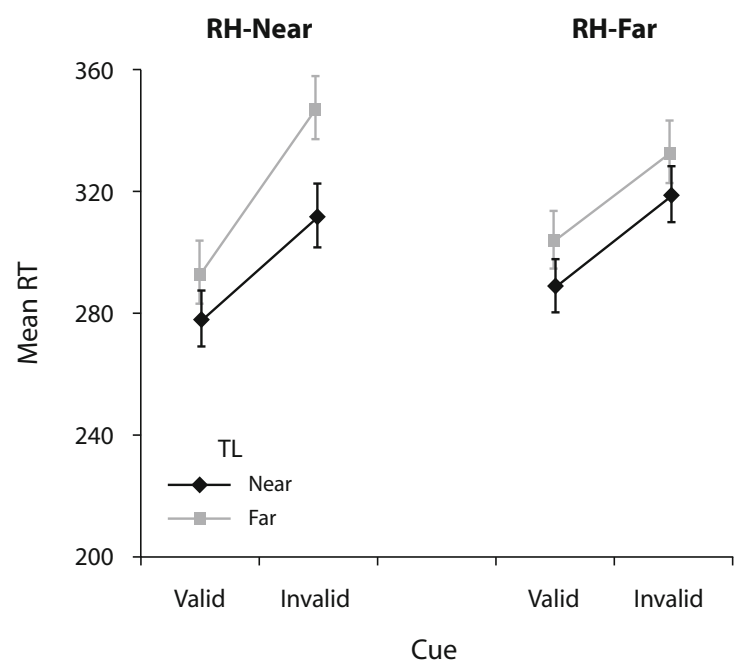

Figure 2. Means of median RTs (in milliseconds), as a function of response hand position (RH-near, RH-far), cue (valid, invalid), and target location (TL) in Experiment 1. 
tively, in the RH-near group, and $275 \mathrm{msec}(S E=7)$ and $289 \mathrm{msec}(S E=7)$, respectively, for the RH-far group. Analysis of the cue control task with the factors cue color (blue, green) and RHP yielded nonsignificant results for cue color $(F<1)$, RHP $(F<1)$, and cue color $\times$ RHP $(F<1)$. Mean RTs to green and blue cue lights presented at the center LED were $234 \mathrm{msec}(S E=5)$ and $236 \mathrm{msec}$ $(S E=6)$ in the RH-near group, and $251 \mathrm{msec}(S E=6)$ and $251 \mathrm{msec}(S E=5)$ in the RH-far group.

To summarize, the analysis of Experiment 1 revealed significant effects of endogenous cuing at $300 \mathrm{msec}$ after presentation of the cue. These effects were characterized by the near effect when the response hand was positioned near the viewer. Specifically, and consistent with previous findings, the effect of invalid cuing on detection times was greater for targets at the far than at the near target location when the hand was positioned at the near target depth. Placing the responding hand at the far depth failed to reverse the near bias to the far target location, suggesting that the reorienting bias observed here and in previous studies was not simply due to the position of the response hand. The finding that the far hand eliminated the asymmetry in cuing effects suggests, however, that spatial attention is influenced by hand position in the depth-cuing task. This effect seems to be restricted to invalid cued targets presented at the far target location. Neither the valid conditions nor the invalid condition at the near target location were affected by the position of the response hand.

Overall, the results of Experiment 1 suggest that reorienting attention to a target beyond fixation is associated with a reduction in detection time cost when the hand is positioned at the far target depth. It remains to be established whether elimination of the near effect is due to the mere presence of the hand at the far location or to the fact that an active manual response had to be made. The possible contribution of a passive hand in the near effect is explored in Experiments 2 and 3. In Experiment 2, we investigate whether the addition of a passive hand in the visual scene will influence the effect of the response hand observed in Experiment 1. Participants were instructed to place one hand on a response key at the near location and the other hand on the key at the far location for the duration of the experiment. Manual responses were made with either the left or the right hand in an alternating fashion across blocks, allowing comparison of passive hand and response hand effects on spatial attention while maintaining the visual scene and proprioceptive information equivalent across conditions. One possible outcome is that the presence or absence of the near effect is dependent on the position of the response hand (consistent with Experiment 1), which would suggest that the hand effect observed in Experiment 1 is predominantly associated with the response requirement, and not with a passive hand. Alternatively, if both the passive hand and the response hand influence shifts of attention, possibly in an additive fashion, the near effect should be (at least partly) reinstated when the passive hand is placed at the near target location, and it should be reduced when the passive hand is placed at the far target location.

\section{EXPERIMENT 2}

\section{Method}

Participants. Sixteen volunteers participated in Experiment 2 (mean age $=21$ years, $S D=3.87$ ). All participants were righthanded, had normal or corrected-to-normal vision, and did not report any problems with color vision or depth perception.

Apparatus and Procedure. The following changes were made to the setup and design for Experiment 2: A second buttonbox (identical to the buttonbox used in Experiment 1) was affixed to the rail. One buttonbox was placed at the far depth, and the other was placed close to the near rod. The positioning of the buttonbox relative to the rod at each depth was identical to the positioning used in Experiment 1. Eight participants placed the right hand on the buttonbox at the far depth and the left hand on the buttonbox at the near depth. This was reversed for the remaining 8 participants. Positioning of the hands on the buttonboxes was the same as that in Experiment 1.

Testing took place in two sessions, each consisting of four blocks of 240 trials. In each block, participants responded either with the right or the left hand. Four of the 8 participants whose left hands were placed at the near depth responded with the left hand in Blocks 1 and 3 of Session 1 and with the right hand in Blocks 1 and 3 in Session 2. This was reversed for the remaining 4 participants. The same counterbalancing procedure was applied to the participants whose right hands were placed in proximity to the near rod.

Participants were instructed to keep the index finger of both the response hand and the passive hand on the response keys for the duration of each block to ensure similarity of proprioceptive information received from both hands before target presentation. Based on the finding that cuing effects were not maximal at the $300-\mathrm{msec}$ SOA in Experiment 1, SOA was increased for Experiment 2. To maximize unpredictability of target onset, SOA was varied at random in steps of $50 \mathrm{msec}$ between 550 and $900 \mathrm{msec}$. The warning tone in the control tasks was removed, and the response-stimulus interval in the target control task was adjusted to the SOA used in the depth-cuing task. The target control task was presented in both sessions: Eight participants responded with the hand positioned at the far location in Session 1 and with the near hand in Session 2. The reverse order was applied for the remaining 8 participants.

\section{Results and Discussion}

Depth-cuing task. Means of median RTs were entered in a mixed, repeated measures ANOVA with the factors response hand position (near, far), target location (near, far), and cue (valid, invalid). The factor hand (either left or right hand placed at the far LED) was entered as the between-participants factor. The results revealed a significant effect of target location $[F(1,14)=32.2, p<.001]$, reflected in longer RTs to far than to near targets, and a significant effect of cue $[F(1,14)=26.7, p<.001]$, with longer RTs to invalid cued targets. Consistent with the results of Experiment 1, cuing effects were greater at the far than at the near $[F(1,14)=8.5, p=.011]$ target location. Post hoc analysis showed that the effect of target location was significant in both cue conditions $(p<.001$, for both comparisons) and that the effect of cue was significant at both target locations ( $p<.001$, for both comparisons). To confirm that asymmetry in the cuing effect was significant, RT difference values were calculated (RT invalid - RT valid). Comparison of these difference values showed that cuing effects were greater at the far than at the near $[t(15)=3.009, p=.009]$ target location. The interaction between target location and RHP was significant $[F(1,15)=9.2, p=.009]$, but this interaction is explained 


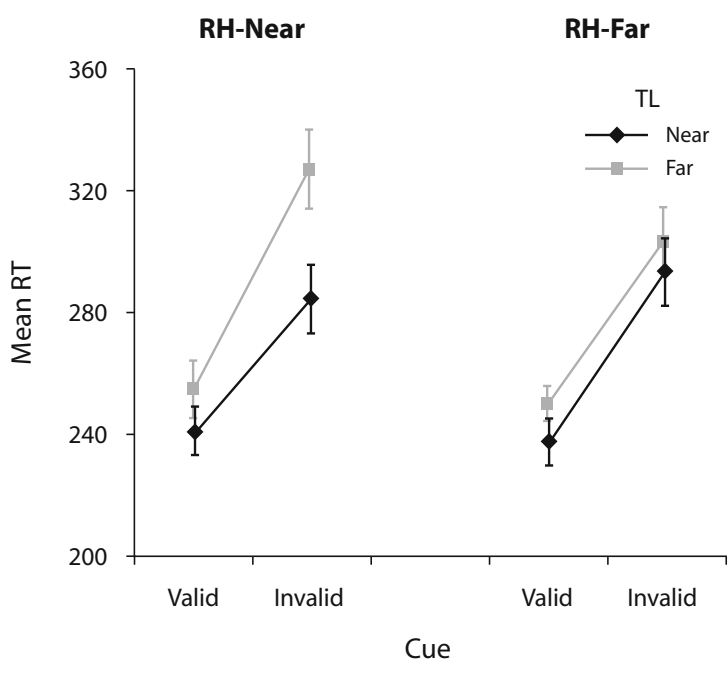

Figure 3. Means of median RTs (in milliseconds), as a function of response hand position (RH-near, RH-far), cue (valid, invalid), and target location (TL) in Experiment 2.

by the more important significant three-way interaction among cue, target location, and $\operatorname{RHP}[F(1,15)=8.06, p=$ $.012]$. Figure 3 shows a pattern of results consistent with the results of Experiment 1: The near effect is present in the RH-near condition, but it is abolished by the response hand when this hand is positioned at the far target location. Post hoc analysis revealed that RTs to invalid cued targets presented at the far target location were significantly faster in the RH-far than in the RH-near ( $p=.006)$ condition, whereas this difference was not significant for invalid cued targets presented at the near target location $(p=.12)$. The difference between RH-far and RH-near conditions was also not significant for valid cued trials $(p>.09)$

Control tasks. The target control task was analyzed using ANOVA with the factors RHP and target location, and the between-participants factor hand (either left or right hand at far depth). This analysis revealed a significant effect of target location $[F(1,14)=51.1$, $p<.001]$, but none of the other effects were significant $(F<1)$. Means of median RTs to near and far targets were $229 \mathrm{msec}(S E=6)$ and $242 \mathrm{msec}(S E=6)$, respectively, in the RH-near condition, and $232 \mathrm{msec}(S E=8)$ and $245 \mathrm{msec}(S E=8)$, respectively, for the RH-far condition. Analysis of the cue control task using ANOVA with the factor cue color (green, blue) and the betweenparticipants factor hand (right, left) revealed nonsignificant results for all effects $(F<1)$. Mean RTs to green and blue cue lights were $218 \mathrm{msec}(S E=5)$ and $221 \mathrm{msec}$ $(S E=5)$, respectively.

To summarize, Experiment 2 revealed the near effect when responses were made with the hand near the viewer, even when a passive hand was positioned at the same depth as the far LED. The finding that the near effect was absent when the response hand was placed at the far target depth, despite there being a passive hand placed at the near depth, suggests that shifts of attention were not affected by the passive hand. The effect of the responding hand at the far target depth was restricted to the condition in which shifts of attention were made from the invalidly cued near location to the far target LED. Consistent with the results of Experiment 1, detection times to valid and invalid cued targets presented at the near location were not affected by RHP. Since the location of both hands was the same for the duration of the testing session in Experiment 2, it is unlikely that the abolishment of the near effect in Experiment 1 was associated with differential attentional saliency effects due to dissimilar visual scenes. Hand dominance was shown not to be a relevant factor either, because the effect of the response hand at the far location was the same for right- and left-handed responses.

The finding that the response hand effect on the reorienting bias was not influenced by the addition of a passive hand does not necessarily show that spatial attention was not influenced by the passive hand. Reed et al. (2006) showed that when attention is cued along the horizontal dimension and only the nonresponding hand is horizontally aligned with one of the two target locations, spatial attention is biased toward this hand's location, resulting in an overall benefit in detection times to the proximal target (independent from cue validity). In Experiment 2 of the present study, detection times to valid targets and to invalid cued targets at the near target location did not vary as a function of passive hand position (PHP). This finding suggests that the passive hand was not prioritized for spatial attention, or alternatively, that the passive hand effect on spatial attention can be revealed only when potentially more dominant effects of the responding hand are eliminated. In Experiment 3, we investigated whether passive hand influences on spatial attention were masked by effects of the response hand in Experiment 2. The nonresponding hand was placed at either the near or the far target depth, while the responding hand was moved away from the target scene. If alignment in depth of the passive hand and the target is sufficient for attention to be prioritized to the passive hand in the depth-cuing task, an overall detection time bias (independent from cue validity) may be revealed at the target location most proximal to the passive hand, in line with the attentional prioritization effect observed in horizontal cuing tasks (Reed et al., 2006).

\section{EXPERIMENT 3}

\section{Method}

Participants. Sixteen participants were recruited for Experiment 3 (mean age $=22$ years, $S D=4.8$ ). All participants were right-handed and had normal or corrected-to-normal vision, without any reported problems with color vision or depth perception.

Procedure. The following modifications to the experimental procedure of Experiment 2 were made for Experiment 3: All participants responded by pressing a response key placed on the table below the rail closer to the viewer (the actual distance between the hand and the near target light was approximately $52 \mathrm{~cm}$, the distance in depth between the hand and the near LED was $17.5 \mathrm{~cm}$, the horizontal distance was $10 \mathrm{~cm}$, and the vertical distance, $50 \mathrm{~cm}$ ). The other hand was placed on either the near or the far buttonbox placed on the rail (depending on the condition), but was never used for responding. Due to malfunction, two LEDs had to be replaced. The luminance 
of the cue lights and target lights was adjusted to $24 \mathrm{~cd} / \mathrm{m}^{2}$ for the green and blue cue lights and $54 \mathrm{~cd} / \mathrm{m}^{2}$ for the red target lights. Eight participants responded with the left hand and placed the right hand on the buttonbox fixed at either the near or far target depth. The remaining 8 participants responded with the right hand and placed the left hand on the buttonbox that was close to either the near or the far LED. The experimental task consisted of four blocks of 240 trials. For 8 participants, the passive hand was placed on the buttonbox at the far target depth during the first two blocks and at the near target depth during the last two blocks. This order was reversed for the remaining 8 participants. The target control task was presented after the first two blocks and at the end of the experimental task.

\section{Results and Discussion}

Depth-cuing task. Means of median RTs were entered in a mixed, repeated measures ANOVA with the factors target location, cue, and PHP (near, far). The factor hand (responses made with either the left or the right hand) was entered as the between-participants factor. Significant main effects were found for the factors cue $[F(1,14)=$ $41.5, p<.001]$ and target location $[F(1,14)=35.9, p<$ $.001]$. The interaction between cue and target location was also significant $[F(1,14)=23.7, p<.001]$. Post hoc analysis showed that the effect of cue was significant at both target locations $(p<.001)$ and that the effect of target location was significant in both cue conditions $(p \leq$ .04). Pairwise comparisons of the difference values (RT invalid - RT valid) as a function of target location showed that the effect of cuing was significantly greater at the far than at the near $[t(15)=5.04, p<.001]$ target location. Most important was the finding that the three-way interaction among cue, target location, and PHP was not significant $(F<1)$, suggesting that the asymmetric cuing effect was comparable in both PHP conditions (see Figure 4).

Control tasks. The target control task was analyzed with the factors PHP (near, far) and target location, and the between-participants factor hand (responding with either the right or the left hand). The effect of target location

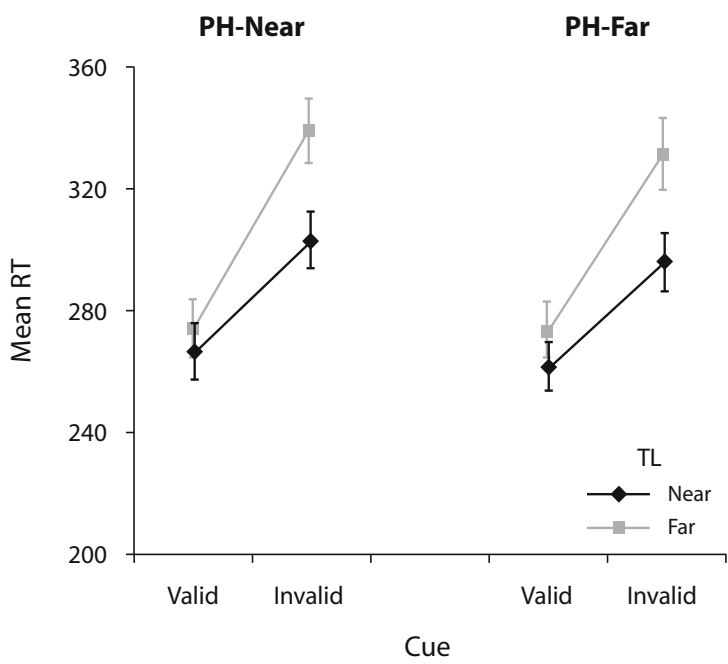

Figure 4. Means of median RTs (in milliseconds), as a function of passive hand position (PH-near, $\mathrm{PH}-\mathrm{far}$ ), cue (valid, invalid), and target location (TL) in Experiment 3. was significant $[F(1,14)=5.7, p=.032]$, but the interaction between target location and PHP was not $(F<1)$. Mean RTs to near and far targets were $248 \mathrm{msec}(S E=8)$ and $260 \mathrm{msec}(S E=9)$, respectively, in the PH-near condition and $244 \mathrm{msec}(S E=8)$ and $254 \mathrm{msec}(S E=7)$, respectively, in the $\mathrm{PH}-$ far condition. The effects of PHP $[F(1,14)=2.1, p=.16]$, hand $(F<1)$, and PHP $\times$ hand $(F<1)$ were not significant, suggesting that neither hand dominance nor the position of the passive hand influenced detection times in the target control task. Analysis of the cue control task with the factors cue light and response hand revealed nonsignificant results for all three effects $($ all $F \mathrm{~s}<1)$. Mean RTs to green and blue cue lights were $222 \mathrm{msec}(S E=7)$ and $223 \mathrm{msec}(S E=6)$, respectively.

Further analysis. The difference between RTs to valid far and valid near targets was consistent across all three experiments, irrespective of response hand or passive hand position. The assumption was that this difference would be associated with the different sizes of the retinal regions covered by the near and far target lights (approximately twice as large for the near light), as suggested by the effect of target location in the target control task. To establish whether the target location effect observed on valid trials in the depth-cuing task was comparable to the effect of target location in the target control task, RTs to valid cued trials and to targets in the target control task were analyzed with the factors target location (near, far) and task (valid cued condition in the depth-cuing task, control task), separately for each experiment. Significant results were found for the effect of target location in all three experiments $(F \geq$ $10.39, p \leq .006)$ and for the effect of task in Experiments 1 and 3 ( $F \geq 7.8, p \leq .013$ ) (faster RTs to targets in the control task), but the latter effect was not significant in Experiment $2[F(1,15)=3.51, p=.081]$. Most important was the finding that the interaction between task and target location was not significant ( $F<1$ in all three experiments), suggesting that differences in detection times for near and far targets were the same in the target control task and on valid cued trials in the depth-cuing task.

A final analysis on RT was conducted to explore whether the cuing effect and the near effect (cue $\times$ target location) were comparable in all conditions in which the response hand was placed near the viewer, whether a passive hand was placed in proximity to the far target LED (Experiments 2 and 3 ) or not (Experiment 1). ANOVA was used with the factors cue and target location. The factor experiment was entered as the between-participants factor. The results of this analysis revealed significant main effects of target location $[F(1,43)=77, p<.001]$ and cue $[F(1,43)$, $p<.001]$, and a significant effect of cue $\times$ target location $[F(1,43)=36.7, p<.001]$, consistent with the main analyses per experiment. The main effect of experiment was not significant $[F(1,43)=1.8, p=.17]$, and the interaction among experiment, cue, and target location was also not significant $[F(1,43)=1.6, p=.21]$. The latter result suggests that the passive hand at the far location did not modulate the near bias in the reorienting of attention when the response hand was placed near the viewer.

The percentages of trials rejected due to the amplitude cutoff were $5.92 \%$ and $6.12 \%$ in the response-hand far 
conditions (for Experiments 1 and 2, respectively), and $5.45 \%$ and $6 \%$ in the response-hand near conditions (Experiments 1 and 2, respectively). For Experiment 3, the percentages of trials rejected were $4.76 \%$ and $5.32 \%$ for the passive hand far and near conditions, respectively. Omissions and anticipatory errors were made infrequently (less than 2\% across all conditions in all three experiments). Because the variance in several conditions was 0 or close to 0 for all three types of errors, only main effects were analyzed for each experiment. Analysis of percentages of eye movement trials, omissions, and anticipations revealed nonsignificant results for all comparisons in all three experiments ( $t \leq 1.5, p \geq 0.14)$.

In sum, placement of only a passive hand at either the near or the far target depth did not affect detection times in Experiment 3. It is therefore unlikely that the absence of possible attentional prioritization effects associated with the passive hand were somehow masked by the more dominant response hand effects in Experiment 2. The presence of the near effect in both PHP conditions supports the idea that the response requirement of the hand at the far target location is crucial for the abolishment of the reorienting bias to occur. Irrelevance of PHP for shifts of attention in the present study is further supported by analysis across experiments, showing that the near effect was not significantly influenced by the presence (Experiments 2 and 3) or absence (Experiment 1) of a passive hand at the far depth when the response hand was placed near the viewer.

RTs to near targets were consistently faster than those to targets emitted from the far LED in the depth-cuing task. Although this difference could be interpreted as a general viewer-centered bias in endogenous shifts of attention to all targets presented in viewer-centered near space, it is more likely that the effect is associated with the different retinal sizes of the two stimuli. The target location effect observed on valid cued trials was comparable to the location effect in the control task, in which endogenous shifts of attention were not required. Further support for this assumption is implied by Gawryszewski et al. (1987), who adjusted the size of the target lights for distance from the viewer in a depth-cuing task and found no location effect in RTs to valid cued targets. Both findings seem inconsistent with the idea that voluntary shifts of attention may be biased to all targets near the viewer.

\section{GENERAL DISCUSSION}

The aim of the present study was to investigate whether the near effect, previously associated with a $3-\mathrm{D}$ viewercentered reorienting bias to near space, could be explained in terms of a bias to the responding hand, always positioned near the viewer in previous studies that investigated spatial attention in real depth situations. The results of Experiment 1 excluded this explanation; the near effect was not reversed to a far effect when the hand was positioned at the far depth. Instead, the hand reduced detection time cost associated with reorienting of attention from the near to the far target location, resulting in symmetric effects of cuing at the near and far LEDs. Shifting attention in re- sponse to valid depth cues was left unaffected by response hand position, as was the reorienting of attention from the far to the near target location. Experiments 2 and 3 further showed that the response requirement was crucial for the hand effect to occur. Placement of only a passive hand at one of the target locations had no effect on detection times (Experiment 3), and placing a passive hand at the target depth not occupied by the responding hand did not influence the effect of the response hand on the reorienting bias (Experiment 2).

One aspect of our results that seems inconsistent with those of Reed et al. (2006), who cued attention along the horizontal dimension, is the absence of any passive hand position effect on detection times in the depth-cuing task. Although this could suggest that attentional prioritization of the passive hand is restricted to situations in which attention is cued along the horizontal dimension, an alternative explanation is that this inconsistency is related to the difference in proximity between the passive hand and the target stimulus in the two studies. The passive hand was proximal to the target along all three dimensions in Reed et al.'s study, whereas the vertical distance between the response key and the LED was $9 \mathrm{~cm}$ in the depth-cuing task used here. Reed et al. showed that the attentional prioritization effect depends on close proximity of the target to the hand (because the effect was not observed when the distance was $11 \mathrm{~cm})$, consistent with the idea that the attentional prioritization effect reflects modulation of spatial attention within a bimodal (visual-tactile), hand-centered spatial representation (Duhamel, Colby, \& Goldberg, 1998; Fogassi et al., 1996; Graziano \& Gross, 1995). Whether an overall bias in spatial attention to the passive hand would have been detected if the hand had been more proximal to the target cannot be excluded. It remains clear, however, that the position of the passive hand cannot account for the presence or absence of the near effect in the depth-cuing task.

Although the present findings clearly suggest that the response requirement is a necessary condition for hand position effects to occur in the depth-cuing task, it is less clear whether spatial attention was modulated within a 3 -D viewer-centered or hand-centered spatial representation. Gawryszewski et al. (1987) originally explained the near effect as reflecting 3-D viewer-centered enhancement of spatial attention in the region between the viewer and fixation after the cuing of the near LED, resulting in prolonged detection time when reorienting of attention to the far target location is required. When the far LED is cued, the attentional focus is shifted to the far target location, and attention spreads toward the viewer, resulting in equal awareness of all information within this viewer-centered area of space. One explanation within this framework for the abolishment of the near effect is that the 3 -D viewercentered attentional space is extended to the depth of the far response hand on all trials during response preparation, even when attention is initially cued to the near LED. This would result in equal awareness of both target locations when the target is presented, and it could account for the symmetric cuing effects when the hand is positioned at the far depth. An alternative explanation for the response hand effect is that a hand-centered spatial representation 
is accessed when one prepares the manual response in addition to an already active 3-D viewer-centered representation. When the hand is placed at the far position and attention is cued to the near LED, response preparation may enhance spatial awareness around the hand to a level equal to that of the attentional space created within a 3-D viewer-centered spatial representation between the viewer and fixation. Assuming that the attended region around the hand includes the target LED, the pattern of results across the two hand position conditions would reflect additive influences of viewer-centered and hand-centered spatial representations on spatial attention in this explanation.

Whereas both explanations could account for the abolishment of the near effect by the response hand at the far target depth, it can be argued that the two explanations would predict different results on valid cued trials. Consistent with the observed pattern of results, the 3-D viewer-centered explanation would predict that valid cue conditions should be left unaffected by hand position, because the cued locations always fall within attended space, regardless of whether the hand is positioned close to the near or the far LED. In contrast, if spatial attention is enhanced within both spatial representations, additive effects of the response hand would be expected in all conditions, including valid cued trials. The only caveat would be if spatial attention were maximally enhanced within a viewer-centered spatial representation in terms of observable behavioral effects. A few neuropsychological findings have previously been explained in this way (e.g., Frassinetti, Rossi, \& Làdavas, 2001; Mattingley et al., 1998). Mattingley et al. showed, for example, that spatial awareness of stimuli presented in right space was not further enhanced by right-handed, trial-initiating keypresses in a patient with left visual extinction. Although spatial awareness in these studies was predominantly measured in terms of the number of stimuli reported, detection times may have been similarly restricted in their sensitivity to uncover additional effects of the response hand. This question clearly requires further investigation, although additive effects in attention (e.g., locationbased and object-based attention) have been successfully revealed within the third dimension using manual RTs as the behavioral measure (Bourke et al., 2006).

Although the specific pattern of results observed in the present study seems most consistent with a 3-D viewercentered explanation for the abolishment of the near effect, this preliminary conclusion may warrant further verification in future studies, particularly given the accumulating evidence for modulation of attention from hand-centered representations in peripersonal space. One question raised by the present findings is whether the beneficial effect of the response hand at the far target depth is restricted to situations in which the response hand is aligned with the direction of the viewer's gaze. It may be possible that the boundary of 3-D viewer-centered enhancement in spatial attention returns to fixation when the response hand at the far target depth is moved farther away from gaze direction. When the hand is positioned close to a target in the periphery, proprioceptive information received from the hand and limb may become more relevant for determin- ing the location of the hand for shifts of attention to the response hand, previously associated with response preparation (Eimer et al., 2005; Eimer \& Van Velzen, 2006). Adding LEDs to the left and right could allow investigation of the relative importance of these two types of information about hand position. The potential influence of a (bimodal) hand-centered representation on shift of attention may result in horizontal biases when the hand is placed in the periphery, instead of (or possibly in addition to) a 3-D viewer-centered reorienting bias to near space.

A second question that could be investigated further is whether the influence of hand-centered representations on spatial attention could be manipulated by the complexity of the manual response. Studies investigating the effect of reaching responses on spatial attention have shown that reaching seems to be associated with action-based shifts of attention to the starting position of the hand (Tipper, Lortie, \& Baylis, 1992). In a study investigating distractor interference on reaching response times, Tipper et al. showed that RT interference was strongest from distractors presented at locations in the middle and front of the 3-D target display when the starting position was close to the observer. This effect reversed when the hand was positioned beyond all stimuli, with stronger interference from distractors farthest from the viewer but closest to the starting position of the reaching hand. Interference from distractors close to the hand have also been shown to affect reaching trajectories (e.g., deviating away from the distractors), supporting the assumption that distractor inhibition operates on representations centered on the hand in goal-directed reaching (Tipper, Howard, \& Jackson, 1997). Perhaps a similar action-based bias to the far hand could be revealed when the behavioral response requires movement planning in the depth-cuing task.

In conclusion, the results of this study demonstrate that the near effect observed in the depth-cuing task can be eliminated by placing a response hand close to the far target location. The hand influenced spatial attention only when it was used for responding (and not when it was passively placed at the far target depth), so the abolishment of the near effect seems to be associated with the preparation of the manual response, resulting in equal spatial awareness of the near and far target location when the hand is positioned at the far depth. The pattern of results across the three experiments is most consistent with the idea that spatial attention can be modulated by the response hand within a 3-D viewer-centered spatial representation when the hand is aligned with the direction of gaze.

\section{AUTHOR NOTE}

Correspondence concerning this article should be addressed to P. M. J. Pollux, Department of Psychology, University of Lincoln, Brayford Pool, Lincoln LN6 7TS, England (e-mail: ppollux@lincoln.ac.uk).

$$
\text { Note-Accepted by the editorial board }
$$
of Editor-Elect Jeremy M. Wolfe.

\section{REFERENCES}

Andersen, G. J. (1990). Focused attention in three-dimensional space. Perception \& Psychophysics, 47, 112-120.

AtChley, P., \& Kramer, A. F. (1998). Spatial cuing in a stereoscopic 
display: Attention remains "depth-aware" with age. Journals of Gerontology, 53B, 318-323.

Atchley, P., Kramer, A. F., Andersen, G. J., \& Theeuwes, J. (1997) Spatial cuing in a stereoscopic display: Evidence for a "depth-aware" attentional focus. Psychonomic Bulletin \& Review, 4, 524-529.

Bourke, P. A., Partridge, H., \& Pollux, P. M. J. (2006). Additive effects of inhibiting attention to objects and locations in three-dimensional displays. Visual Cognition, 13, 643-654.

Couyoumdian, A., Di Nocera, F., \& Ferlazzo, F. (2003). Functional representation of 3D space in endogenous attention shifts. Quarterly Journal of Experimental Psychology, 56A, 155-183.

Downing, C. J., \& PINKER, S. (1985). The spatial structure of visual attention. In M. I. Posner \& O. S. M. Marin (Eds.), Attention and performance XI: Mechanisms of attention (pp. 171-187). Hillsdale, NJ: Erlbaum.

Duhamel, J.-R., Colby, C. L., \& Goldberg, M. E. (1998). Ventral intraparietal area of the macaque: Congruent visual and somatic response properties. Journal of Neurophysiology, 79, 126-136.

Eimer, M., Forster, B., Van Velzen, J., \& Prabhu, G. (2005). Covert manual response preparation triggers attentional shifts: ERP evidence for the premotor theory of attention. Neuropsychologia, 43, 957-966.

EIMER, M., \& VAN VELZEN, J. (2006). Covert manual response preparation triggers attentional modulations of visual but not auditory processing. Clinical Neurophysiology, 117, 1063-1074.

Fogassi, L., Gallese, V., Fadiga, L., Luppino, G., Matelli, M., \& Rizzolatti, G. (1996). Coding of peripersonal space in inferior premotor cortex (area F4). Journal of Neurophysiology, 76, 141-157.

Frassinetti, F., Rossi, M., \& LÀdavas, E. (2001). Passive limb movements improve visual neglect. Neuropsychologia, 39, 725-733.

Gawryszewsi, L. D. G., Riggio, L., Rizzolatti, G., \& Umiltà, C. (1987). Movements of attention in the three spatial dimensions and the meaning of "neutral" cues. Neuropsychologia, 25, 19-29.

Graziano, M. S. A., \& Gross, C. G. (1995). The representation of extrapersonal space: A possible role for bimodal, visual-tactile neurons. In
M. S. Gazzaniga (Ed.), The cognitive neurosciences (pp. 1021-1034). Cambridge, MA: MIT Press.

Han, S., Wan, X., \& Humphreys, G. W. (2005). Shifts of spatial attention in perceived 3-D space. Quarterly Journal of Experimental Psychology, 58A, 753-764.

Jonides, J., \& MACK, R. (1984). On the cost and benefit of cost and benefit. Psychological Bulletin, 96, 29-44.

Mattingley, J. B., Robertson, I. H., \& Driver, J. (1998). Modulation of covert visual attention by hand movement: Evidence from parietal extinction after right-hemisphere damage. Neurocase, 4, 245-253.

Müller, H. J., \& RabbitT, P. M. A. (1989). Reflexive and voluntary orienting of visual attention: Time course of activation and resistance to interruption. Journal of Experimental Psychology: Human Perception \& Performance, 15, 315-330.

Parks, N. A., \& Corballis, P. M. (2006). Attending to depth: Electrophysiological evidence for a viewer-centered asymmetry. NeuroReport, 17, 643-647.

Previc, F. H. (1998). The neuropsychology of 3-D space. Psychological Bulletin, 124, 123-164.

Reed, C. L., Grubb, J. D., \& Steele, C. (2006). Hands up: Attentional prioritization of space near the hand. Journal of Experimental Psychology: Human Perception \& Performance, 32, 166-177.

Theeuwes, J., Atchley, P., \& Kramer, A. F. (1998). Attentional control within 3-D space. Journal of Experimental Psychology: Human Perception \& Performance, 24, 1476-1485.

TIPPER, S. P., HowARD, L. A., \& JACKSON, S. R. (1997). Selective reaching to grasp: Evidence for distractor interference effects. Visual Cognition, 4, 1-38.

Tipper, S. P., Lortie, C., \& Baylis, G. C. (1992). Selective reaching: Evidence for action-centered attention. Journal of Experimental Psychology: Human Perception \& Performance, 18, 891-905.

(Manuscript received June 29, 2007; revision accepted for publication April 24, 2008.) 